\title{
Analysis of the Clinicopathologic Characteristics and Prognosis of Head and Neck Lymphoma
}

\author{
Shufang Yan $\left(\mathbb{D},{ }^{1,2}\right.$ Jiajia Ma $\mathbb{D}^{1,2}$ Meihong Yang $\mathbb{D}^{1,2}$ Bo Liu $\mathbb{D},{ }^{1,2}$ Sijing Li $\mathbb{D},{ }^{1,2}$ \\ Liuqing Yang $\mathbb{D}^{1,2}$ Qian Zhang $\mathbb{D}^{1,2}$ and Xinxia Li $\mathbb{D}^{1}$ \\ ${ }^{1}$ Department of Pathology, The Tumor Hospital Affiliated to Xinjiang Medical University, No. 789 Suzhou Dongjie, Urumqi, \\ The Xinjiang Uygur Autonomous Region of China, 830011, China \\ ${ }^{2}$ Xinjiang Medical University, No. 567 North Shangde Road, Urumqi, The Xinjiang Uygur Autonomous Region of China, \\ 830011, China \\ Correspondence should be addressed to Xinxia Li; lxx-patho@163.com
}

Received 11 September 2021; Revised 14 December 2021; Accepted 22 January 2022; Published 22 February 2022

Academic Editor: Consuelo Amantini

Copyright (C) 2022 Shufang Yan et al. This is an open access article distributed under the Creative Commons Attribution License, which permits unrestricted use, distribution, and reproduction in any medium, provided the original work is properly cited.

Statistical reports on non-Hodgkin's lymphoma (NHL) of the head and neck combining clinical medicine with pathology are rare. To provide a basis for prognosis prediction and individualized treatment, we will investigate the clinicopathologic characteristics and prognosis of lymphoma in the head and neck region. Four hundred sixty-one patients with NHL in the head and neck region diagnosed through histological biopsy were retrospectively analyzed. Fluorescence in situ hybridization (FISH) and immunohistochemistry (IHC) were performed in all cases to evaluate the genetic status and protein expression levels. Patients were followed up by telephone. The prevalence rate of primary extranodal NHL (PENHL) in the head and neck region was 44.62\% (166/372). The incidence of extranodal lymphoma accounted for 36.66\% (169/461) of all head and neck lymphomas. Among the cases of PENHL of the head and neck, diffuse large B-cell lymphoma (DLBCL) (60/76, 78.95\%) and extranodal NK/T-cell lymphoma, nasal type (ENKTCL) (21/24, 87.5\%) were the most common subtypes originating from B-cell lymphoma (BCL) and T-cell lymphoma (TCL), respectively. The most common sites of nodal and extranodal onset were neck lymph nodes and the gastrointestinal tract, respectively. The most common and primary locations of BCL and TCL were the tonsils and nasal cavity, respectively. The 3-year survival rates of PENHL, ENKTCL, and DLBCL of the head and neck were $42 \%, 28.57 \%$, and $41.67 \%$, respectively, and the 5-year survival rates were $24 \%, 19.05 \%$, and $20 \%$, respectively. Survival analysis showed that male sex was a risk factor $(\mathrm{HR}=5.421 ; 95 \% \mathrm{CI}, 1.164-25.267 ; p<0.05)$ and that comprehensive treatment was a protective factor $(\mathrm{HR}=0.117 ; 95 \% \mathrm{CI}, 0.025-0.545 ; p<0.05)$ against extranodal DLBCL in the head and neck region. Bone marrow involvement was a risk factor for PENHL of the head and neck (HR $=5.072 ; 95 \% \mathrm{CI}, 1.17-21.991 ; p<0.05)$. The purpose of this review is to show that PENHL of the head and neck with high incidence deserves more attention, and a model of multidisciplinary diagnosis and treatment should be adopted.

\section{Introduction}

Lymphoma is a frequent hematological malignancy. In China, lymphoma has the highest incidence of the hemolymphatic system [1]. According to histopathology, lymphoma can be separated into two major categories: Hodgkin's lymphoma (HL) and non-Hodgkin's lymphoma (NHL). The subtypes of lymphoma are defined in accordance with the origin of the cells: B-cell lymphoma (BCL), T-cell (TCL) and natural killer-cell lymphoid tumors (T/NK-NHL), and
HL [2]. NHL accounts for approximately $90 \%$ of all lymphomas [3]. Approximately $30 \%$ of NHLs originate from extranodal sites. Among the primary extranodal non-Hodgkin's lymphomas (PENHLs), the head and neck are often involved [2], and extranodal manifestations of head and neck lymphomas are different in the head and neck region and may occur in the major salivary glands, paranasal sinuses, mandible, maxilla, and Waldeyer's ring (largely depending on the specific NHL subtypes and frequently with their own characteristics). Judging by this, NHL of the head and neck region is a 
common disease in the process of clinicopathologic diagnosis. Due to a lack of adequate knowledge about this kind of disease and other reasons, it is often difficult to diagnose, and clinicians tend to miss it or misdiagnose it. In previous literature from home and abroad, most related studies on NHL of the head and neck are clinical analyses, but statistical reports on clinical medicine and pathology are rare. In our study, we reviewed epidemiological data, the clinical and pathological characteristics, treatment, and prognosis of the disease. The main purpose of this study was to summarize the incidence and features of extranodal NHL in the head and neck region from a single center in western China to provide a reference for the diagnosis and treatment of NHL.

\section{Material and Methods}

2.1. Clinical Data. A standardized medical history was obtained from all patients, including clinical examination results, age at diagnosis, sex, ethnicity, specific location of NHL in the head and neck, imaging evaluation (CT or B ultrasound), vital symptoms (B-symptoms: fever, night sweats, and weight loss), initial diagnosis time, known risk factors (infectious diseases such as HIV, hepatitis B, hepatitis C, tuberculosis, syphilis, and other systemic diseases, such as hypertension, diabetes, arrhythmia, and coronary atherosclerotic heart disease), histological biopsy results, and survival results. All patients finished the clinical examination (laboratory examination and histological biopsy) and underwent B-mode color Doppler ultrasonography of the neck when necessary. Some patients also underwent plain and enhanced CT scans of the head, neck, chest, abdomen, and pelvic cavity and a bone marrow biopsy. Blood tests included a complete blood count, and the following related parameters were recorded: white blood cell count, platelet count, and hemoglobin. Ann Arbor staging was performed in 100 patients with PENHL of the head and neck. Immunophenotyping based on Han's classification was used for patients diagnosed with diffuse large B-cell lymphoma (DLBCL). The aaIPI score and IPI score were determined according to age ( $\leq 60$ years old and $>60$ years old), and pathological parameters such as the expression of proteins, rearrangement of genes, and positivity for Epstein-Barr virus (EBV) were recorded. During the diagnosis, all patients also underwent enhanced CT scans of the head, neck, chest, abdomen, and pelvic cavity or PET-CT and bone marrow biopsies. If central nervous system involvement was suspected, Ann Arbor staging also included contrast magnetic resonance imaging of the brain and/or spine. According to the 2013 Chinese guidelines for the diagnosis and treatment of DLBCL, the efficacy of the disease is divided into four stages: $\mathrm{CR}-$ complete remission, $\mathrm{PR}-$ partial remission, $\mathrm{PD}-$ progressive disease, and $\mathrm{SD}$-stable disease.

\subsection{Pathological Data}

2.2.1. Immunohistochemistry (IHC). Using a fully automatic immunohistochemical instrument, antibodies suitable for paraffin sections were chosen. The main antibodies used and their working concentrations are shown in Table 1.
Among them, the cutoff scores for MYC and Bcl-2 overexpression were $\geq 40 \%$ and $\geq 70 \%$, respectively, and the positive standards for CD10, Bcl-6, and MUM1 were $\geq 30 \%$ [4]. Double-expressor lymphoma (DEL) refers to simultaneous expression of $\mathrm{MYC} / \mathrm{Bcl}-2$ protein (DEL-MYC/Bcl2 ), and triple-expressor lymphoma (TEL) refers to simultaneous expression of $\mathrm{MYC} / \mathrm{Bcl}-6 / \mathrm{Bcl}-2$ protein (TEL-MYC/ $\mathrm{Bcl}-2 / \mathrm{Bcl}-6)$ [5] in lymphoma patients. DLBCL was classified according to Han's algorithm, where the germinal center B cell (GCB) subtype had a CD10+ or CD10-, Bcl-6+, and MUM1- phenotype, whereas the Bcl-6- or Bcl-6+, CD10-, and MUM1+ phenotype represented the non-GCB subtype (including $\mathrm{ABC}$ subtype and unclassified subtype in this study).

2.3. Fluorescence In Situ Hybridization (FISH). FISH was performed on 60 DLBCL formalin-fixed paraffin-embedded (FFPE) samples following the manufacturer's instructions. Detection of MYC, Bcl-2, and Bcl-6 gene abnormalities and two-color separation and rearrangement probe (including Bcl-6, MYC, and Bcl-2) kits were purchased from the Abbott Molecular Biology Company. Samples with MYC, Bcl-2, and Bcl-6 segregation were used as positive controls, and normal lymph nodes were used as negative controls. The FISH signal pattern of the separation probe in normal cells consists of two yellow signals or tightly located red (the organ color shows red on the purple background of DAPI) and green signals, and the distance between them is less than two signal sizes. Cells with a yellow, green, and red signal with a distance of $\geq 2$ signal sizes were defined as positive for gene rearrangement, with three separate probes. The probe signals for a monolayer of at least 200 tumor cell nuclei were counted per sample at 100x magnification, and genetic alterations were determined when they exceeded a $10 \%$ threshold in the number of nuclei. FISH V.2.0 software was used to analyze the results.

2.4. In Situ Hybridization. An EBER (EBV-encoded RNA) in situ hybridization kit was purchased from ZSBIO. The specific steps were performed according to the manufacturer's instructions, and nasopharyngeal carcinoma tissues with positive EBER were treated as a positive control.

2.5. Follow-Up Visits. The time of diagnosis was when the follow-up visits began, and the deadline was August 19, 2020. The patient follow-up visits were held at the hospital or via telephone. The reasons for termination of the follow-up (such as death due to illness, loss to follow-up, or other irrelevant reasons) were recorded. Total survival time (TST) was defined as the time from the date of pathological diagnosis to the time of death or last follow-up. The median survival time (MST) is also known as the half-life, and a cumulative survival rate of 0.5 is the corresponding survival time. Overall survival (OS) is the ratio of the total number of survivors at the end of the entire follow-up period to the total number of people initially observed. The survival time of the patients who died within 1 month after the initial diagnosis was recorded as " 0 ." 
TABle 1: Primary antibodies and conditions used for immunohistochemical staining.

\begin{tabular}{|c|c|c|c|c|}
\hline Antigen & Clone & Source & Dilution & Positive location \\
\hline ALK & SP8 & ZSBIO & $1: 80$ & Nuclei/cytoplasm \\
\hline Bcl-2 & 100/D5 & Shanghai Long Island & $1: 100$ & Cytoplasm \\
\hline Bcl-6 & GI191E/A8 & ZSBIO & $1: 80$ & Nuclei \\
\hline CD3 & UCHT1 & Shanghai Long Island & $1: 50$ & Cytoplasm/cytomembrane \\
\hline CD5 & SP19 & ZSBIO & $1: 100$ & Cytomembrane \\
\hline $\mathrm{CD} 8$ & SP16 & ZSBIO & $1: 100$ & Cytomembrane \\
\hline CD10 & $56 \mathrm{C} 6$ & Gene Company & $1: 50$ & Cytomembrane \\
\hline $\mathrm{CD} 20$ & L26 & Dako & $1: 150$ & Cytomembrane \\
\hline CD30 & Ber-H2 & MXBIO & $1: 100$ & Cytomembrane/cytoplasm \\
\hline $\mathrm{CD} 43$ & DF-T1 & ZSBIO & $1: 100$ & Cytomembrane/cytoplasm \\
\hline CD45RO & UCHL1 & Dako & $1: 100$ & Cytomembrane \\
\hline CD56 & IB6 & Gene Company & $1: 100$ & Cytomembrane \\
\hline CD79a & HM47/A9 & Shanghai Long Island & $1: 100$ & Cytomembrane \\
\hline Cyclin D1 & SP4 & Gene Company & $1: 80$ & Nuclei \\
\hline EMA & GP1.4 & Shanghai Long Island & $1: 200$ & Cytomembrane \\
\hline Granzyme-B & GZB01 & MXBIO & $1: 50$ & Cytoplasm \\
\hline MUM1 & Mum1p & MXBIO & Ready to use & Nuclei \\
\hline MYC & Y69 & ZSBIO & $1: 150$ & Nuclei \\
\hline Ki67 & MIB-1 & Gene Company & $1: 150$ & Nuclei \\
\hline Perforin & MRQ-23 & ZSBIO & $1: 20$ & Cytoplasm \\
\hline TIA-1 & 2G910F5 & ZSBIO & $1: 100$ & Cytoplasm \\
\hline
\end{tabular}

2.6. Statistical Analysis. The experimental data were processed using SPSS 23.0 statistical software. Unpaired $t$ tests were used for measurement data, and differences between different groups were compared using the $\chi^{2}$ test or Fisher's exact test. Survival curves were drawn according to the Kaplan-Meier method in univariate survival analysis, and the log-rank test was used to compare survival rates among various groups. A Cox regression model was applied to multivariate survival analysis, and the bilateral 95\% CI was treated as the statistical standard. A $p$ value of $<0.05$ was considered statistically significant.

\section{Results}

3.1. Epidemiology and Characteristics of the Head and Neck Cohort. One thousand twenty-nine patients with lymphoma diagnosed through histological biopsy at the Tumor Hospital Affiliated with Xinjiang Medical University from January 2010 to July 2020 were retrospectively analyzed, including 128 HL (extranodal: 118; nodal: 10) and 901 NHL (extranodal: 559; nodal: 342) (Figure 1(a)). The most common sites of nodal onset and extranodal onset were neck lymph nodes and the gastrointestinal tract, respectively. In this study, for the first time, 461 patients with head and neck lymphomas diagnosed by histological biopsy were retrospectively analyzed. The number of patients with lymphadenopathy was 292, and the incidence of extranodal disease was 169 , accounting for $63.34 \%(292 / 461)$ and $36.66 \%(169 / 461)$ of head and neck lymphomas, respectively (Figure 1(b)). Finally, 89 cases of HL were screened: the number of extra- nodal patients was 3 , and the number of nodal patients was 86. Three hundred seventy-two cases of NHL were selected: extranodal: 166, 44.62\%; nodal: 206, $55.38 \%$ (Figure 1(c)). Among the 372 NHL patients, DLBCL accounted for 172 cases: seventy-five extranodal patients were included, and 97 nodal patients were included in this study, accounting for $43.61 \%$ and $56.39 \%$ of all DLBCL cases, respectively. Among the 166 extranodal NHL patients, 66 cases of NHL were removed due to incomplete extranodal NHL data; finally, 100 cases were included in this study. Among the 100 patients with primary extranodal NHL in the head and neck region, 59 were males and 41 were females, aged between 2 and 87 years; the median age was 58 , and 58 cases were $\geq 50$ years old. There were 5 cases of bone marrow involvement and 21 cases of multiple site involvement. This study included 67 Han nationalities and 33 ethnic minorities.

Among the 100 patients with primary extranodal NHL in the head and neck region (Figure 1(d)), 76 cases of Bcell lymphoma were identified: sixty cases of DLBCL, six cases of mucosa-associated lymphoid tissue extranodal marginal zone lymphoma (MALT), three cases of follicular lymphoma (FL), three cases of Burkitt lymphoma (BL), one case of B small lymphoid cell lymphoma/chronic lymphocytic leukemia, two cases of B-lymphoblastic lymphoma (BLBL), and one case of mantle cell lymphoma. DLBCL was the most common subtype with a median age of 58 and an average age of 55.88. Twenty-four cases of T-cell lymphoma were identified: twenty-one cases of extranodal NK/T cell lymphoma, nasal type (ENKTCL); two cases of peripheral 


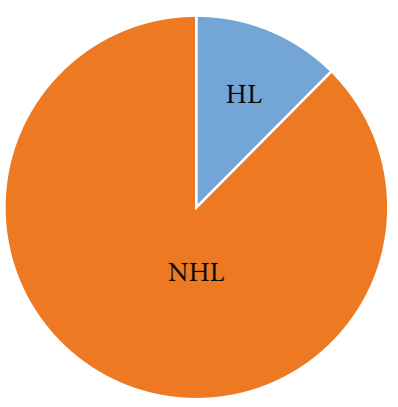

(a)

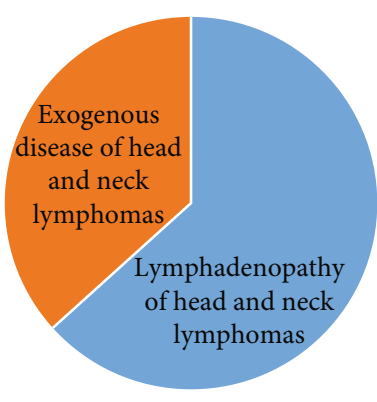

(b)

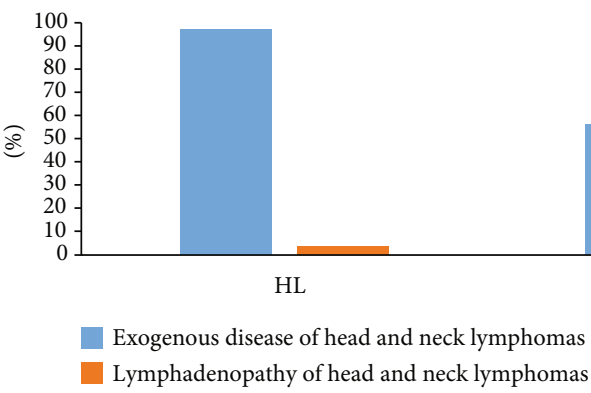

(c)

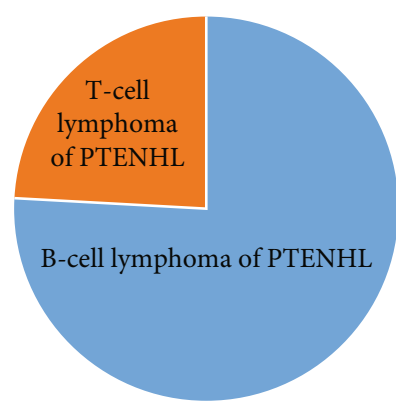

(d)

Figure 1: Clinical characteristics of extranodal non-Hodgkin's lymphoma in the head and neck region: (a) proportion of all lymphomas; (b) lymphadenopathy or exogenous disease proportion of head and neck lymphoma; $(c, d)$ two major categories and two subtypes of PENHL in the head and neck region. PENHL: primary extranodal non-Hodgkin's lymphoma; HL: Hodgkin's lymphoma; NHL: non-Hodgkin's lymphoma.

T-cell lymphoma (PTCL); and one case of anaplastic large cell lymphoma (ALCL) with positive ALK. ENKTCL accounted for the highest ratio, which included 13 males and 8 females; the ratio of males to females was 1.6 , and the median age was 49 . The most common locations of primary BCL and TCL were the tonsils and nasal cavities, respectively (Table 2 ).

Among the 31 patients with NHL originating from the nose, 10 cases arose from the nasopharynx, whereas the remaining cases involved the nasal cavity. There were 17 cases of primary ENKTCL, three cases of DLBCL, and one case of PTCL. Twenty-seven patients had B-symptoms, 16 patients had abnormal hemograms, 28 patients had no recorded International Prognostic Index (IPI) score, five patients received IPI scores but did not have improved IPIs, and nine patients were still incomplete after rescoring (Table 2).

3.2. Immunophenotypic Features. BCL: sixty cases of DLBCL were identified. The expression rates of CD10, Bcl-6, CD20, Mum1, Ki67 index $\geq 70 \%$, Bcl-2, and MYC were $18.33 \%$ (Figure 2(a)), 41.67\% (Figure 2(b)), 100\%, 60\% (Figure 2(c)), $45 \%$ (Figure 2(d)), 20\% (Figure 2(e)), and 18.33\% (Figure 2(f )), respectively. There were 23 cases of GCB type, 34 cases of $A B C$ type, and three cases of unclassified DLBCL. Six cases of DE lymphoma and five cases of TE lymphoma were included. Six cases of MALT were identified: the expression rates of $\mathrm{CD} 20, \mathrm{CD} 43, \mathrm{PAX} 5$, and $\mathrm{Bcl}-2$ were $100 \%, 25 \%$, $60 \%$, and $100 \%$, respectively. Three cases of FL were identified: the expression rates of $\mathrm{Bcl}-2$ and $\mathrm{CD} 20$ were $66.67 \%$ and $100 \%$, respectively. Three cases of BL were identified: the expression rates of Bcl-6, CD10, CD20, CD79a, and Ki67 index $\geq 70 \%$ were $100 \%$. Two cases of B-LBL were identified: the expression rates of CD10, CD20, CD79a, PAX5, and TdT were 100\%. One was B small lymphoid cell lymphoma/ chronic lymphocytic leukemia: the expression rates of CD5, CD23, CD20, and CD79a were 100\%, and the Ki67 index was $\geq 40 \%$. One case of MCL was identified: the expression rates of Cyclin D1, CD5, CD20, and CD79a were 100\%, and the Ki67 index was $\geq 60 \%$.

TCL: twenty-one cases of ENKTCL were identified. The expression rates of EBER, CD20, CD79a, CD56, CD3, CD4, CD8, GrB1, TIA-1, CD43, and Ki67 $\geq 60 \%$ were $100 \%$ (Figure 3(a)), 5.88\%, 42.86\%, 61.11\% (Figure 3(b)), 100\% (Figure 3(c)), 71.43\%, 60\%, 75\% (Figure 3(d)), 93.75\%, $66.67 \%$, and $28.57 \%$ (Figure 3(e)), respectively. One case was ALCL with ALK positivity: the expression rates of ALK, CD30, CD2, CD3, and EMA were $100 \%$. Two cases of PTCL were identified: the expression rates of CD3 (Figure 4(a)) and Ki67 (Figure 4(b)) index $\geq 70 \%$ were $100 \%$. The expression rates of CD20 (Figure 4(c)) and CD23 (Figure 4(d)) were negative.

In the above 100 cases, due to incomplete tests of MYC, Bcl-2, or Bcl-6, DE and TE could not be acquired completely.

3.3. Cytogenetic Analysis. Out of the 60 patients with DLBCL, 33 finished $\mathrm{TH}$ or $\mathrm{DH}$, no patient was confirmed with $\mathrm{TH}$, and two patients accounting for $3.33 \%(2 / 60)$ of the patients with DLBCL had double-hit lymphoma (DHL). All of them had DHL-MYC/Bcl-6, including 1 case of DHL combined with TEL (Figures 5(a)-5(c)). 
TABLE 2: Frequency of histologic types of head and neck lymphoma including epidemiology, symptomatology, disease manifestations, localization, and laboratory findings.

\begin{tabular}{|c|c|c|c|}
\hline Item & B-cell lymphoma & T-cell lymphoma & $p$ value \\
\hline$n$ & 76 & 24 & - \\
\hline Age & - & - & 0.186 \\
\hline Mean \pm SD & $54.37 \pm 19.59$ & $48.54 \pm 15.45$ & - \\
\hline Median & 58 & 48 & - \\
\hline Sex, $n[\%]$ & - & - & 0.939 \\
\hline Male & $45[59.21 ; 45]$ & $14[58.3 ; 14]$ & - \\
\hline Female & $31[40.78 ; 31]$ & $10[41.7 ; 10]$ & - \\
\hline Ethnicity, $n$ [\%] & & & 0.591 \\
\hline Han & $52[68.42 ; 52]$ & $15[62.5 ; 15]$ & - \\
\hline Minorities & $24[31.58 ; 24]$ & $9[37.5 ; 9]$ & - \\
\hline Primary localization, $n$ [\%] & - & - & $0.000 *$ \\
\hline Nasopharynx & $8[10.53 ; 8]$ & $2[8.33 ; 2]$ & - \\
\hline Nasal cavity & $4[5.26 ; 4]$ & $17[70.83 ; 17]$ & - \\
\hline Tonsil & $22[28.95 ; 22]$ & $0[0]$ & - \\
\hline Mandible & $14[18.42 ; 14]$ & $1[4.17 ; 1]$ & - \\
\hline Parotid gland & $6[7.89 ; 6]$ & $0[0]$ & - \\
\hline Laryngopharynx & $4[5.26 ; 4]$ & $3[12.5 ; 3]$ & - \\
\hline Tongue & $4[5.26 ; 4]$ & $0[0]$ & - \\
\hline Others & $14[18.42 ; 14]$ & $1[4.17 ; 1]$ & - \\
\hline Laboratory parameter, mean \pm SD & - & - & - \\
\hline Leucocytes & $6.84 \pm 3.28$ & $7.18 \pm 5.07$ & 0.697 \\
\hline Hemoglobin & $125.22 \pm 22.30$ & $117.92 \pm 16.86$ & 0.143 \\
\hline Thrombocyte & $236.60 \pm 89.41$ & $236.33 \pm 95.54$ & 0.990 \\
\hline LDH increased & $21[27.63 ; 21]$ & $8[33.33 ; 8]$ & 1.000 \\
\hline Systemic disease, $n$ [\%] & $33[43.42 ; 33]$ & $4[16.67 ; 4]$ & $0.024 *$ \\
\hline Communicable diseases, $n$ [\%] & $9[10.53 ; 8]$ & $1[4.17 ; 1]$ & 0.343 \\
\hline Hepatitis B & $7[9.21 ; 7]$ & $1[4.17 ; 1]$ & 0.427 \\
\hline HIV & $2[2.63 ; 2]$ & $0[0]$ & 0.422 \\
\hline Others & $24[31.58 ; 24]$ & $3[12.50 ; 3]$ & 0.066 \\
\hline Histologic subtype, $n[\%]$ & 76 & 24 & - \\
\hline DLBCL & $60[78.95 ; 60]$ & - & - \\
\hline MALT & $6[7.89 ; 6]$ & - & - \\
\hline FL & $3[3.95 ; 3]$ & - & - \\
\hline $\mathrm{BL}$ & $3[3.95 ; 3]$ & - & - \\
\hline B small lymphoid cell lymphoma/chronic lymphocytic leukemia & $1[1.32 ; 1]$ & - & - \\
\hline B-LBL & $2[2.62 ; 2]$ & - & - \\
\hline MCL & $1[1.32 ; 1]$ & - & - \\
\hline ENKTCL & - & $21[87.50 ; 2]$ & - \\
\hline PTCL & - & $3[12.50 ; 3]$ & - \\
\hline B-symptoms, $n[\%]$ & $16[21.05 ; 16]$ & $11[45.83 ; 11]$ & $0.017 *$ \\
\hline Composition ratio of death, $n$ [\%] & $14[18.42 ; 14]$ & $7[29.17 ; 7]$ & 0.190 \\
\hline DLBCL & $13[17.11 ; 13]$ & - & - \\
\hline ENKTCL & - & $6[25 ; 6]$ & - \\
\hline Others & $1[1.32 ; 1]$ & $1[4.17 ; 1]$ & - \\
\hline
\end{tabular}

Features of explanation: ${ }^{*} p<0.05$ represents statistical differences. GCB subtype: germinal center B-cell subtype; ABC subtype: activated B-cell-like subtype; LDH: lactate dehydrogenase; DLBCL: diffuse large B-cell lymphoma; ENKTCL: extranodal NK/T cell lymphoma, nasal type; PTCL: peripheral T-cell lymphoma; B-LBL: B-lymphoblastic lymphoma; MCL: mantle cell lymphoma; MALT: mucosa-associated lymphoid tissue extranodal marginal zone lymphoma; FL: follicular lymphoma; BL: Burkitt lymphoma. 


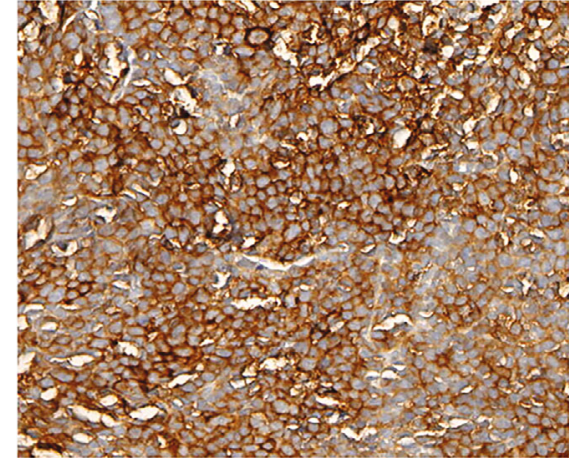

(a)

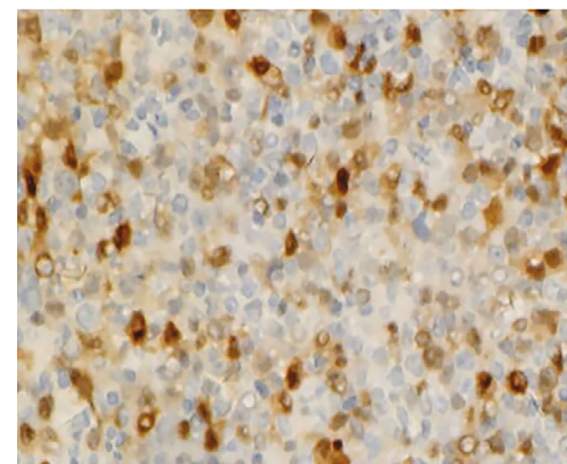

(c)

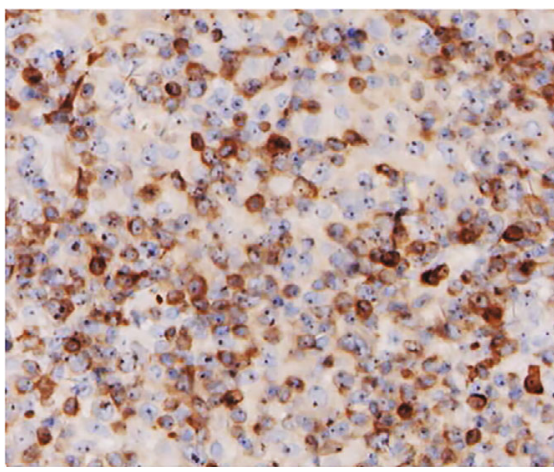

(e)

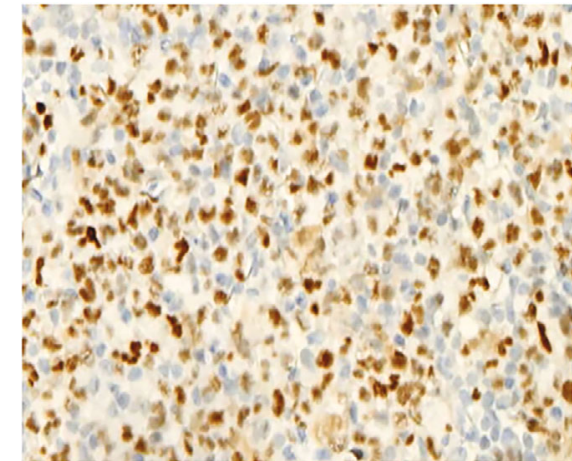

(b)

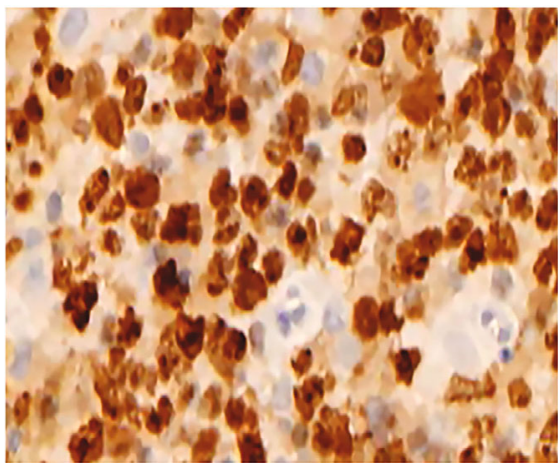

(d)

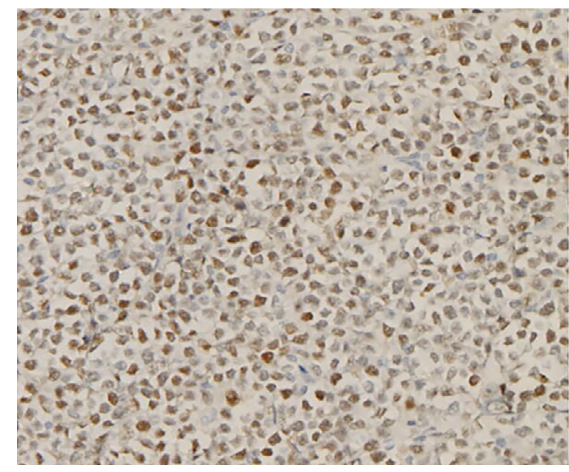

(f)

Figure 2: Immunohistochemical results (EnVision method, original magnification 200x) of DLBCL. (a-f) CD10, Bcl-6, Mum1, Ki67, Bcl-2, and MYC expression on oncocytes.

\subsection{Clinical Outcomes}

3.4.1. Follow-Up and Ann Arbor Staging. During a follow-up of 240 months, 100 patients were followed up systematically. The MST was 24 months ( 1 to 240 months), and the average follow-up time was 37 months. Seven patients were lost to follow-up, and 21 died during the follow-up period. Among the patients with B-cell lymphoma (DLBCL), three died in the early stage (stages I-II), and 10 died in the late stage (stages III-IV). One patient with MALT died in the late stage and lived for 11 months. Among the patients with T-cell lymphoma (ENKTCL), one died in the early stage, and five died in the late stage. During the follow-up, three patients who had been confirmed to be in PR still complained of yellow, pungent pus coming out of their nostrils. One ENKTCL patient with positive CD20 was a 73-year-old elderly male with occasional fatigue complicated with hypertension and $\mathrm{Ki} 67>5 \%$. In this patient, PR was achieved after comprehensive treatment (radiotherapy or chemotherapy or chemoradiotherapy combined with surgery and/or transplantation), TST was 105 months, the original focus was reduced by more than $50 \%$, and no new or metastatic lesions were found in CT reexamination. One patient with EBER-positive PTCL died in the late stage after living for 40 months. The other patient was a middle-aged female with Ki67 > 85\%, a submental primary site, PD after chemotherapy, and TST of 11 months.

\subsection{Treatment Method and Therapeutic Effect Analysis}

3.5.1. Patients with B-Cell Lymphoma. Sixty patients with DLBCL were found: thirty-three patients finished EBV testing: two were positive for EBV, and the MST was 51 months. 


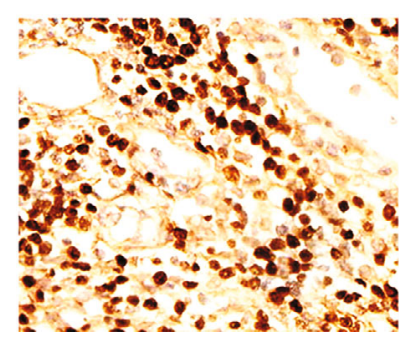

(a)

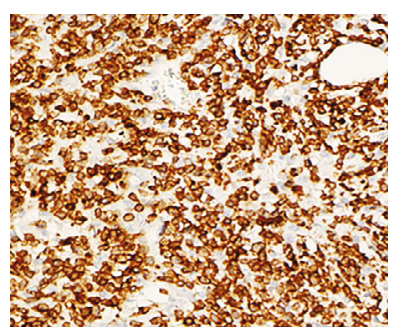

(c)

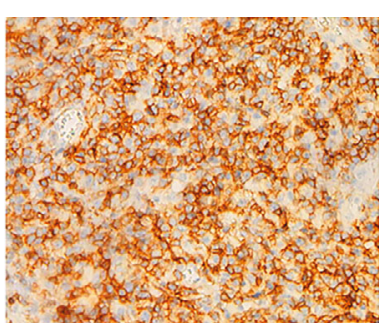

(b)

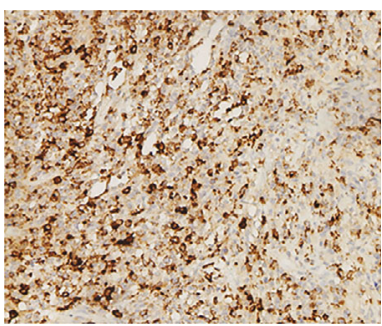

(d)

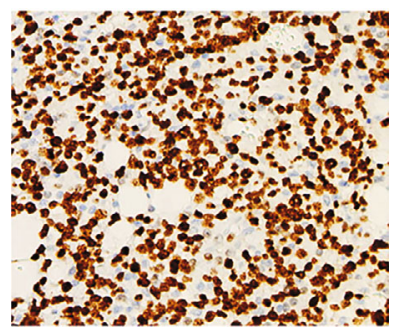

(e)

FIGURE 3: Immunohistochemical results (EnVision method, original magnification 200x) of extranodal NK/T cell lymphoma, nasal type (ENKTCL): (a) EBER-positive oncocytes; (b-e) CD56, CD3, GrB, and Ki67 expression on oncocytes. EBER: Epstein-Barr virus-encoded RNA.

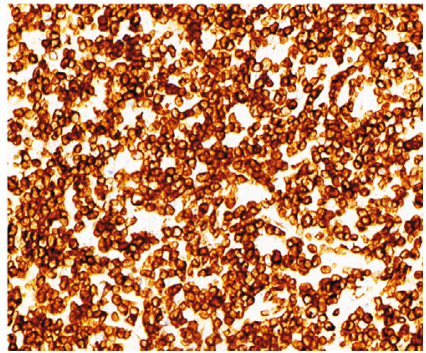

(a)

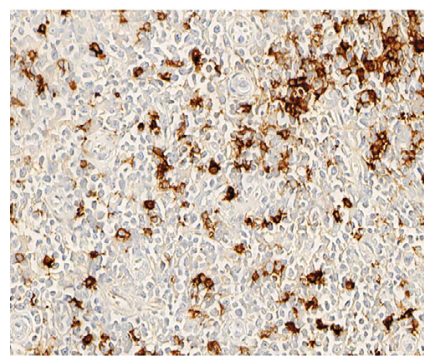

(c)

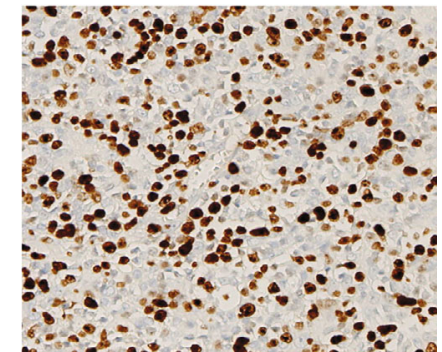

(b)

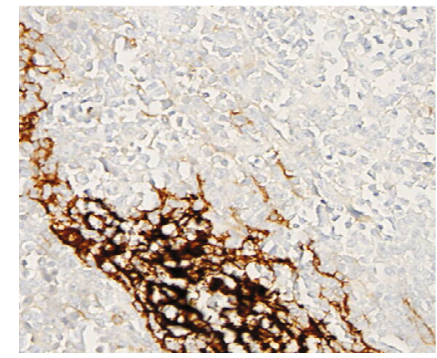

(d)

FIgURe 4: Immunohistochemical results (EnVision method, original magnification 200x) of peripheral T-cell lymphoma: (a, b) CD3 and Ki67 expression on oncocytes; (c, d) CD20- and CD23-negative oncocytes. 


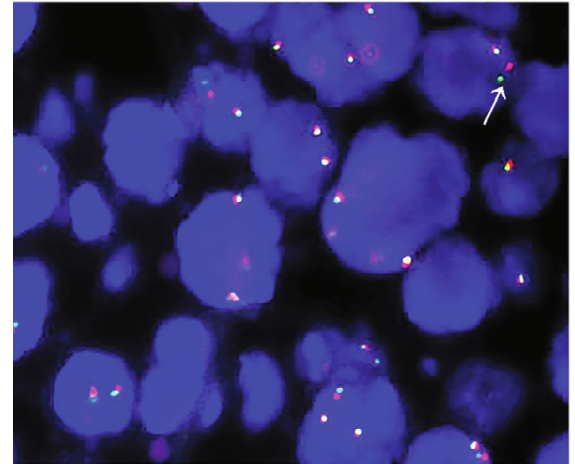

(a)

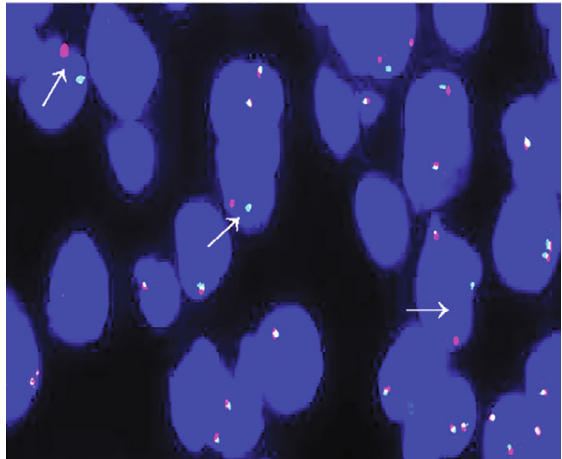

(b)

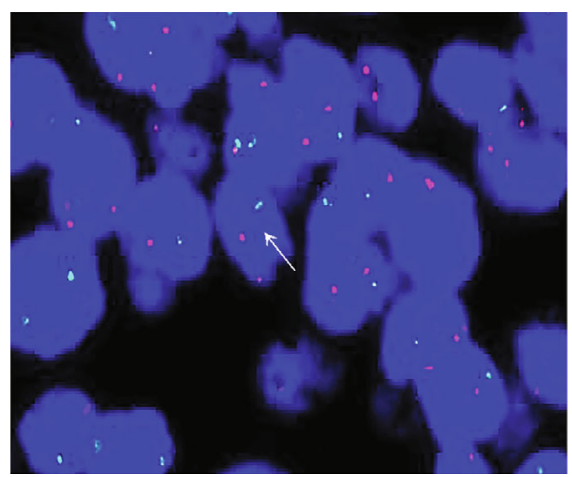

(c)

FIGURE 5: Representative FISH findings in patients with B-cell lymphoma. (a-c) Shows Bcl-2, Bcl-6, and MYC rearrangements. FISH: fluorescence in situ hybridization $(\times 1000)$.

Thirty-one were negative for EBV, and the MST was 24 months. Thirty-four cases of $\mathrm{ABC}$ type were detected: nine patients died, with an MST of 14 months. Four cases were lost to follow-up. Twenty-one cases survived, with an MST of 45.5 months. Twenty-three cases of GCB type were detected: three patients died with an MST of 15 months. Twenty cases survived with an MST of 44 months. Three cases of unclassified DLBCL type were detected: one patient died with a TST of 40 months, and two patients survived. The 3-year and 5-year survival rates of DLBCL were $41.67 \%$ and $20 \%$, respectively, and there was a significant difference in the 3-year and 5year mortality rates $\left(\chi^{2}=6.604, p<0.05\right)$.

Six cases of MALT were detected: five were in early stage and one was in the late stage. One died and five survived. Two patients were complicated with an autoimmune disease called Sjogren's syndrome, and their MST was 32.5 months. To slow down renal dysfunction, one patient with a history of kidney failure used chemotherapy only once, and then, the patient did nothing about kidney failure and lymphoma at the same time. Ultimately, the two diseases reinforced each other, and the patient died after a TST of 40 months; the primary site was located in the nasopharynx. The other one was an elderly female. The primary site was in the parotid gland, she achieved PR after comprehensive treatment, survived until the end of follow-up, and her TST was 25 months. The remaining four survived after treatment. After chemotherapy, two cases with an MST of 35.5 months achieved CR, 3 cases were in the oral cavity. Three cases of FL were found, with a male/female ratio of approximately $1: 1.5$. There was no bone marrow involvement at the initial diagnosis, one was in the early stage, two were in the late stage, the median age was 56 , the average survival time was more than 5 years, 2 achieved CR after chemotherapy, 1 achieved SD after chemotherapy, the MST was 64 months, and all survived at the end of follow-up. One case of B small lymphocytic lymphoma/chronic lymphocytic leukemia was found in an elderly male who had a Ki67 > 40\%, the primary site was the mandible, the TST was 76 months, and PR was achieved after chemotherapy. Three cases of BL were found: a female child, a young male, and an elderly male, with a median age of 29 years; Ki67 was close to $100 \%$; all were in the late stage; and the primary sites were the maxilla, gingiva, and tongue root. Two cases were SD after chemotherapy, one case reached $\mathrm{PR}$, and the MST was 41 months. Two cases of B-LBL were found: all were male children with a median age of 6.5 years, the primary sites were the preauricular position and parotid gland, one achieved PR after chemotherapy, and the other achieved SD after comprehensive treatment. The MST was 41 months. One case of MCL was found in an elderly male with Ki67 $>50 \%$, the primary site was the mandible, the patient achieved CR after comprehensive treatment, and the TST was 95 months.

3.5.2. Patients with TCL. Among 21 patients with ENKTCL, three patients were lost to follow-up, twelve survived, and six 
died. Among the deceased patients, the MST was 9.5 months, one was complicated with hepatitis $\mathrm{B}$, and three cases were located in the nasal cavity and two in the laryngopharynx, of which two patients developed hemophagocytic syndrome and eventually died with pancytopenia and abnormal liver function. One received comprehensive treatment, multiple chemotherapy schemes were changed frequently from the beginning of treatment, the disease progressed continuously, and the TST was 41 months. Another accepted not only continuous chemotherapy but also frequent changes in chemotherapy regimens; owing to the rapid PD, the TST was 12 months. Sixteen patients had completed EBV tests, and all of them had positive results. Five patients did not finish the EBV tests. The number of patients with primary sites in the nasal part and laryngopharynx was 19 and 2, respectively. The 3-year and 5-year survival rates of ENKTCL were $28.57 \%$ and $19.05 \%$, respectively, but there was no difference in the 3-year and 5-year mortality composition $\left(\chi^{2}=0.525, p>0.05\right)$.

One case of ALCL with positive ALK was a young male with Ki67 > 90\%, the primary site was the mandible, CR was achieved after chemotherapy, and the TST was 8 months. One case of PTCL was seen: the patient was a young female with a primary site of the nasal cavity who achieved CR after comprehensive treatment. The TST was 240 months. She was 20 years old and survived until the end of follow-up. One case of PTCL was seen: a middle-aged female with the primary site of the chin, who died after a follow-up of 11 months.

A significant difference was seen in the positive rate of EBV between the ENKTCL and DLBCL groups $\left(\chi^{2}=\right.$ $65.304, p \leq 0.001)$. For the ENKTCL patients, the 3 -year and 5-year survival rates were shorter than those of the DLBCL patients, but there was no difference in the 3-year $\left(\chi^{2}=1.129, p>0.05\right)$ and 5-year mortality rates $\left(\chi^{2}=0.009\right.$, $p>0.05)$. The 3-year and 5-year survival rates of primary extranodal NHL in the head and neck region were $42 \%$ and $24 \%$, respectively, and there was a significant difference in the 3 -year and 5 -year mortality rates $\left(\chi^{2}=7.327, p<0.05\right)$.

\subsection{Survival Analysis}

3.6.1. Univariate Survival Analysis. Among the DLBCL patients, the prognosis of patients with the GCB type was better than that of patients with the non-GCB subtype $\left(\chi^{2}=2.127, p>0.05\right)$ (Figure 6(a)). Comprehensive treatment and operation or chemotherapy were related to the OS of DLBCL patients $\left(\chi^{2}=7.885, p<0.05\right)$ (Figure 6(b)). Sex, age greater than or equal to 50 years, ethnicity, multiple site involvement, bone marrow involvement, primary site, combined systemic disease, and increased $\mathrm{LDH}$ and $\mathrm{B}-$ symptoms were not related to the OS of DLBCL patients. For patients with ENKTCL, bone marrow involvement $\left(\chi^{2}=20.000, p \leq 0.001\right)$ (Figure 6(c)) and increased LDH $\left(\chi^{2}=14.492, p \leq 0.001\right)$ (Figure $\left.6(\mathrm{~d})\right)$ were related to OS. The OS of patients with positive EBV was lower than that of negative patients $\left(\chi^{2}=4.691, p<0.05\right)$ (Figure 6(e)). When comprehensive treatment was applied, the OS of patients with DLBCL was higher than that of ENKTCL patients $\left(\chi^{2}=5.400, p<0.05\right)($ Figure $6(\mathrm{f}))$. There was no difference in OS between patients with B-cell lymphoma and T-cell lymphoma $\left(\chi^{2}=2.053, p>0.05\right)$ (Figure 6(g)). There was no difference in OS between patients with DLBCL and ENKTCL $\left(\chi^{2}=1.358, p>0.05\right)$ (Figure 6(h)). In the head and neck region, there was no difference in OS between patients with DLBCL and PENHL $\left(\chi^{2}=0.028, p>0.05\right)$, and no difference was seen between patients with ENKTCL and PENHL $\left(\chi^{2}=1.365, p>0.05\right)$.

3.6.2. COX Multivariate Survival Analysis. Male sex was a risk factor $(\mathrm{HR}=5.421 ; 95 \% \mathrm{CI}, 1.164-25.267 ; p<0.05)$, and comprehensive treatment was a protective factor $(\mathrm{HR}=0.117 ; 95 \% \mathrm{CI}, 0.025-0.545 ; p<0.05)$ for extranodal DLBCL in the head and neck region. Bone marrow involvement was a risk factor for extranodal NHL in the head and neck region $(\mathrm{HR}=5.072 ; 95 \% \mathrm{CI}, 1.17-21.991 ; p<0.05)$.

\section{Discussion}

Lymphoma is the third most frequent malignancy in the world, accounting for $3 \%$ of all malignant tumors. Lymphoma accounts for $12 \%$ of all malignant tumors of the head and neck and is the third largest malignant tumor after squamous cell carcinoma and thyroid cancer $[2,6,7]$. In recent years, the incidence of NHL in China has tended to advance $[8,9]$. This study shows that in the past 20 years, the extranodal incidence of all head and neck lymphomas was 36.66\% $(169 / 461)$, which was lower than that reported $(57 \%$ and $50 \%$, respectively) [2]. The most common site of onset was the neck lymph nodes, followed by the gastrointestinal tract, which was consistent with a previous report [10]. This may be related to some patients who go to other hospitals in this area. The distribution of extranodal and nodal lymphomas and the primary sites of lymphomas was consistent with previous research [10]. Consistent with a previous study [11, 12], the prognosis of patients with early-stage disease was better than that of patients with late-stage disease, and improving the detection of EBV and making a clear diagnosis in time were beneficial to patients. This may be combined with systemic disease and explains why DLBCL patients have higher mortality. When the patient was a male with DLBCL or a patient was diagnosed with NHL combined with bone marrow involvement, it indicated a poor prognosis.

4.1. Patients with BCL. Consistent with the literature [13, 14], DLBCL accounted for the largest proportion of extranodal NHL of the head and neck. DLBCL can occur in patients at any age, but the majority of them are middle-aged and elderly patients, which is consistent with the results of this study, and the incidence of extranodal disease represents approximately $40 \%$ [15], a result that was similar to that of this study. MYC, Bcl-2, and/or Bcl-6 rearrangement and protein expression were identified as prognostic factors for DLBCL $[16,17]$. This study indicates that immunohistochemistry plays a vital role in forecasting prognosis. Pathologists should improve DEL, TEL, and FISH examinations as soon as possible to discover high-grade B-cell lymphoma with poor prognosis. The number of cases of THL was lower 


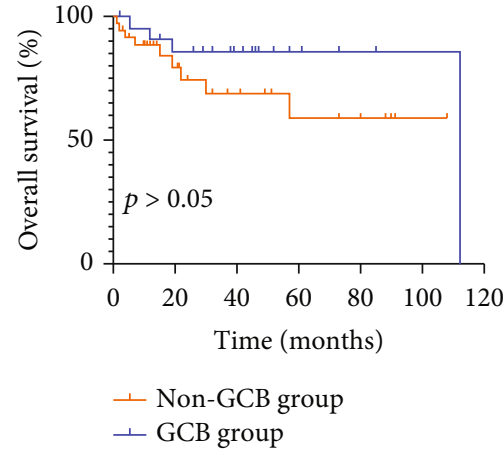

(a)

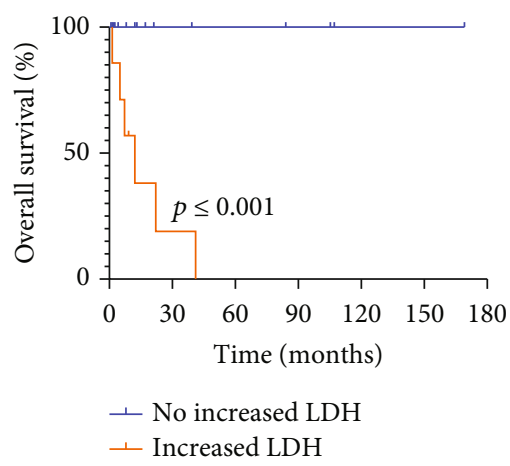

(d)

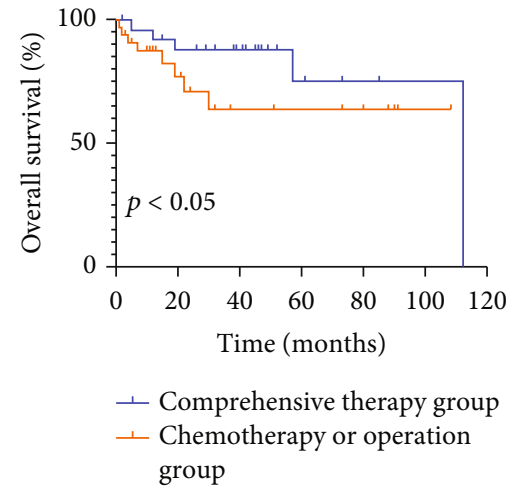

(b)

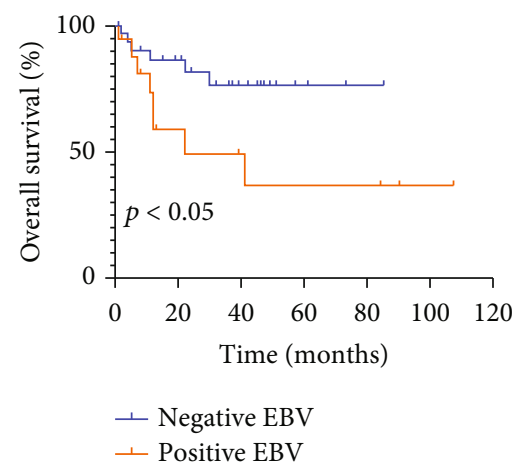

(e)

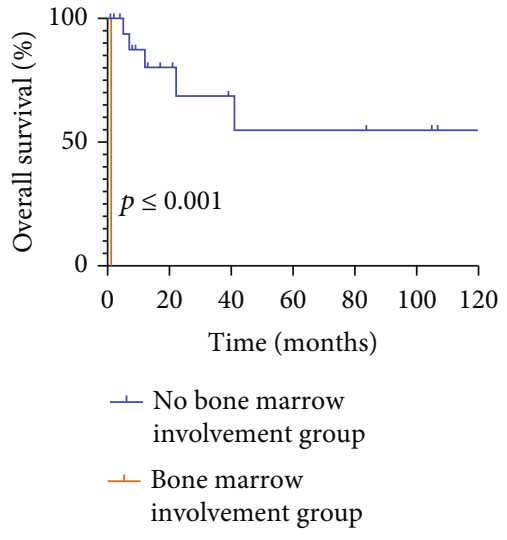

(c)

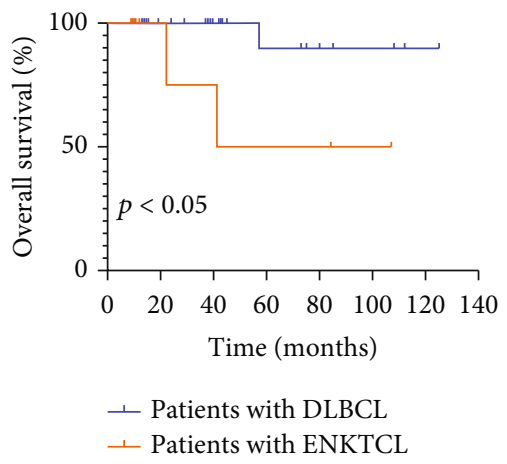

(f)

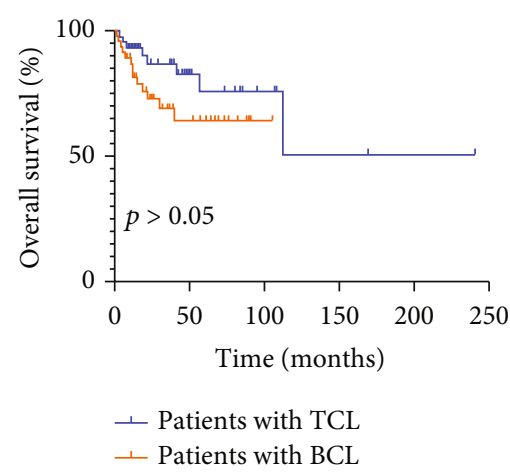

(g)

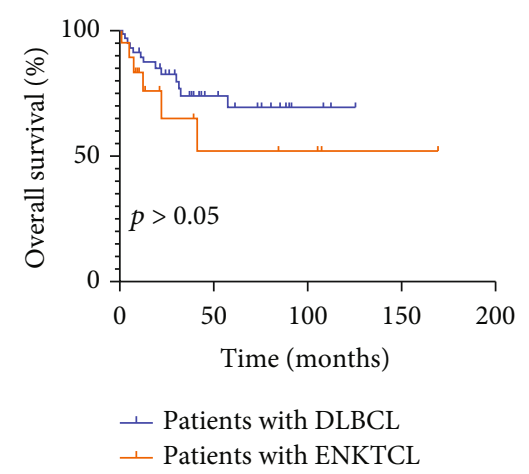

(h)

FIgURE 6: Univariate analysis of patients with primary extranodal non-Hodgkin's lymphoma in the head and neck region among various groups. (a) The prognosis of patients with GCB type was better than that of patients with non-GCB subtype. (b) Comprehensive treatment and operation or chemotherapy was related to the OS of DLBCL patients. (c, d) For patients with ENKTCL, bone marrow involvement and increased LDH were related to OS. (e) The OS of patients with positive EBV was lower than that of the negative patients. (f) When comprehensive treatment was applied, the OS of patients with DLBCL was higher than that of patients with ENKTCL. (g) There was no difference in OS between patients with T-cell lymphoma and B-cell lymphoma. (h) There was no difference in OS between patients with DLBCL and ENKTCL. LDH: lactate dehydrogenase; ENKTCL: extranodal NK/T-cell lymphoma, nasal type; DLBCL: diffuse large B-cell lymphoma.

and the number of cases of DHL was higher than reported [4]. It may be associated with the short prefix time of the specimen, which was applied so that the protease was easy to digest and the hybridization site was fully exposed. Clinical studies have shown that EBV-positive DLBCL is considered to be a new NHL, representing $30-40 \%$ of cases of NHL
[18]. Considering the number of cases, the MST was longer than that of EBV-negative cases, which was inconsistent with the literature [12]. Consistent with a previous report [19], patients with non-GCB type have a worse prognosis than those with GCB type. Regarding age and DLBCL prognosis, continuous controversy has existed $[20,21]$. Perhaps 
for Chinese patients, the age of initial diagnosis over 60 years old will indicate a poor prognosis, which may be related to the Chinese diet, physique, heredity, and other factors. These findings need to be confirmed by further research. During our follow-up, we found that although some patients underwent autologous hematopoietic stem cell transplantation (ASCT) in later stages, the effect of final treatment was poor because the best time for transplantation was missed. Whether sex and B-symptoms are related to the TST of DLBCL still needs to be studied using multicenter and large samples. The 3-year survival rates were lower than those reported in the literature, which may be related to completely resected tumors [22]. MALT can be further divided into gastrointestinal MALT and nongastrointestinal MALT. In the early stage, this lesion is called "pseudolymphoma" [23]. Two cases were complicated with Sjogren's syndrome, which was consistent with a previous report [24]. Five survived until the end of follow-up, and the disease appeared in different places, which once again confirmed that MALT develops slowly and is an inert lymphoma that can occur in many sites. The MALT type is a rare extranodal NHL subtype in the head and neck region, and it mainly occurs in the oral cavity, which is consistent with a previous report [25]. FL is a malignant lymphoma originating from B-cells in the follicular germinal center with an upward trend. A median age of 56 and a male-to-female ratio of $1: 2$ were consistent with the results of a related study [26]. An average survival time of more than 5 years was consistent with a previous research [27]. No case included partial transformation to DLBCL and bone marrow involvement, which was inconsistent with a previous report [28], considering fewer cases. BL is a kind of NHL originating from GCB. The Ki67 being close to $100 \%$ is consistent with this study [29]. Additionally, this study showed that BL was diagnosed initially at a higher stage, which was easily overlooked, and we should pay more attention to it to choose an appropriate treatment strategy to improve the therapeutic effect. BL is classified as endemic, sporadic, and immunodeficient [15]. The primary sites, which were the maxilla, gingiva, and tongue root, were not consistent with those reported [30]. After chemotherapy, all patients survived until the end of follow-up, and the expression of proteins was consistent with that mentioned in a previous report [31].

According to this study, the majority of BCLs in the head and neck regions are DLBCLs. We should place importance on DLBCL. Therefore, doctors in hematology, oncology, and pathology departments should collaborate with each other in their day-to-day work and try their best to advocate for ASCT in time to improve the prognosis. On the other hand, the diagnosis and treatment of lymphoma require multidisciplinary cooperation.

4.2. Patients with TCL. In China, ENKTCL is one of the most common subtypes of NHL [32] and is closely related to EBV infection [33]. EBV infection present in the occurrence of ENKTCL was confirmed once again. The 3-year and 5-year survival rates were lower than those reported in previous reports $[34,35]$. Some ignored the seriousness of refusing to accept treatment at the initial stage, which then resulted in increasing mortality. Consistent with previous reports $[15,36]$, the most common site of ENKTCL and the primary site with the highest incidence of NHL were the nasal cavity. The histologic type with the highest incidence was DLBCL, followed by ENKTCL, which was not consistent with a previous report [36]. Xinjiang is a multiethnic region, and the above result may be related to the fact that there were 33 minority cases. Ethnic and individual differences may lead to a high incidence of DLBCL. In this group of ENKTCL patients, the mortality rate and MST were lower than those reported in the literature [35], so attention must be paid to these patients. Two patients developed hemophagocytic syndrome: one received comprehensive treatment, 1 received chemotherapy, and 2 died of rapid PD. Therefore, when the patient has hemophagocytic syndrome, the condition is critical, the prognosis is extremely bad, and the mortality is high. The positive rate of CD56 was $61.11 \%$, which is a lower ratio than that reported [37], considering the lower number of cases of ENKTCL in this group. Consistent with a report [38], one patient with CD20 positivity in this group was a 73-year-old male, but inconsistent with that report, the $\mathrm{Ki} 67$ was $>5 \%$, and there was low invasiveness and inert clinical progress, as well as accompanying hypertension and occasional fatigue. Increased LDH and bone marrow involvement relevant to OS of ENKTCL were consistent with those reported in a previous study [39].

This study suggests that clinicians usually pay attention to screening EBV-positive patients. In combination with assessing the clinical symptoms, further examination should be recommended if necessary to help doctors exclude ENKTCL as soon as possible. Focusing on the hemogram may mislead the clinician. Previously, patients' chief complaints and medical histories were vital. Observing the dynamics of disease development is indispensable. Biopsy surgeons should strive to obtain a sufficient amount of pathological tissue. Additionally, if there are no clear tumor cells in the first biopsy but a large area of coagulative and necrotic tissue can be seen, it is necessary to perform another tissue biopsy or close follow-up. Fast and accurate pathological diagnosis is a required condition to improve the effect and prolong the lifespan of patients with ENKTCL.

$\mathrm{B}$-symptom is the main prognostic factor [35]. In this study, 27 patients had B-symptoms, which was higher than previously reported [40]. Weight loss, which is relatively subjective, may result in false-positives for B-symptoms. Considering the problem of credibility, clinicians should not judge the disease only by B-symptoms, which may mislead the doctor. Only one patient suffering from HIV was diagnosed with DLBCL, which was inconsistent with a previous report [41]. Among the BL patients who were found, two were males, as reported [42], and none of the cases were associated with HIV or EBV, which was inconsistent with the report [41]. Furthermore, consistent with previous reports, PTCL and B small lymphocytic lymphoma/chronic lymphocyte leukemia were discovered [13, 31]. MCL [43] and lymphoblastic lymphoma [31] are rare subtypes. It has been reported that plasmoblastoma can be seen, but it was not seen in this study [31]. 


\section{Conclusions}

This study showed that the incidence of primary extranodal NHL in the head and neck region was high. Simultaneously, we were not able to apply methods that are useful for guiding clinical diagnosis and treatment until we knew more about the pathology, such as the results of the FISH and IHC tests. To control the progression of NHL in the head and neck, new research methods for tumor prevention, diagnosis, and treatment are necessary. This study reminds otolaryngologists, hematologists, and oncologists to combine clinical disciplines with pathology and pathophysiology, which is beneficial for doctors to understand the pathological and pathophysiological mechanisms of the disease and may be the next step for clinicians and pathologists to explore.

\section{Data Availability}

All data generated or analyzed during this study are included in this published article.

\section{Ethical Approval}

This retrospective study was approved by the Medical Ethics Committee of the Tumor Hospital Affiliated with Xinjiang Medical University (20160218-13).

\section{Consent}

All patients and/or their families signed informed consent forms before histological biopsy, which was carried out by the patients' chief physician. The informed consent forms were filed in the patients' medical record files, and we could not obtain them. Because we were eager to study the specimens, our study did not require the signing of informed consent forms again because only their data were analyzed in our study.

\section{Conflicts of Interest}

The authors declare that they have no conflicts of interest.

\section{Authors' Contributions}

SFY and XXL conceived and designed the study. JJM, MHY, BL, SJL, LQY, and QZ performed the study and analyzed the data. SFY wrote the manuscript. All authors contributed equally to the manuscript and read and approved the final version of the manuscript.

\section{Acknowledgments}

This research was funded by the project of Cisco-Roche Tumor Research Foundation (No. Y-Roche 2019/2-0061) and the project of National Natural Science Foundation of China under Grant No. 81660036.

\section{References}

[1] Hematology Oncology Committee of China Anti-cancer Association, Leukemia \& Lymphoma Group, Society of Hematology at Chinese Medical Association, Chinese Union of lymphoma Research, Chinese Society of Clinical Oncology, "The Chinese expert consensus on hematopoietic stem cell transplantation for malignant lymphoma," Chinese Journal of Clinical Oncology, vol. 40, p. 927, 2018.

[2] K. Storck, M. Brandstetter, U. Keller, and A. Knopf, "Clinical presentation and characteristics of lymphoma in the head and neck region," Head \& Face Medicine, vol. 15, no. 1, pp. 1-6, 2019.

[3] J. O. Armitage, R. D. Gascoyne, M. A. Lunning, and F. Cavalli, "Non-Hodgkin lymphoma," Lancet, vol. 390, no. 10091, pp. 298-310, 2017.

[4] S. Huang, L. Nong, W. Wang et al., "Prognostic impact of diffuse large B-cell lymphoma with extra copies of MYC, BCL2 and/or BCL6: comparison with double/triple hit lymphoma and double expressor lymphoma," Diagnostic Pathology, vol. 14, no. 1, p. 81, 2019.

[5] C. Y. Ting, K. M. Chang, J. W. Kuan et al., "Clinical significance of BCL2, C-MYC, and BCL6 genetic abnormalities, Epstein-Barr virus infection, CD5 protein expression, germinal center B cell/non-germinal center B-cell subtypes, coexpression of MYC/BCL2 proteins and co-expression of MYC/BCL2/BCL6 proteins in diffuse large B-cell lymphoma: a clinical and pathological correlation study of 120 patients," International Journal of Medical Sciences, vol. 16, no. 4, pp. 556-566, 2019.

[6] J. S. Cooper, K. Porter, K. Mallin et al., "National Cancer Database report on cancer of the head and neck: 10-year update," Head \& Neck, vol. 31, no. 6, pp. 748-758, 2009.

[7] W. Hiddemann, D. L. Longo, B. Coiffier et al., "Lymphoma classification-the gap between biology and clinical management is closing," Blood, vol. 88, no. 11, pp. 4085-4089, 1996.

[8] L. A. Torre, F. Bray, R. L. Siegel, J. Ferlay, J. Lortet-Tieulent, and A. Jemal, "Global cancer statistics, 2012," CA: a Cancer Journal for Clinicians, vol. 65, no. 2, pp. 87-108, 2015.

[9] W. Chen, R. Zheng, P. D. Baade et al., "Cancer statistics in China, 2015," CA: a Cancer Journal for Clinicians, vol. 66, no. 2, pp. 115-132, 2016.

[10] J. Sun, Q. Yang, Z. Lu et al., "Distribution of lymphoid neoplasms in China," American Journal of Clinical Pathology, vol. 138, no. 3, pp. 429-434, 2012.

[11] Z. Ma, J. Niu, Y. Cao et al., "Clinical significance of 'double-hit' and 'double expression' lymphomas," Clinical Pathology, vol. 15, p. 206199, 2020.

[12] T. Oyama, K. Yamamoto, N. Asano et al., "Age-related EBVassociated B-cell lymphoproliferative disorders constitute a distinct clinicopathologic group: a study of 96 patients," Clinical Cancer Research, vol. 13, no. 17, pp. 5124-5132, 2007.

[13] H. S. Chi, K. W. Lee, F. Y. Chiang et al., "Head and neck extranodal lymphoma in a single institute: a 17 -year retrospective analysis," The Kaohsiung Journal of Medical Sciences, vol. 28, no. 8, pp. 435-441, 2012.

[14] S. Etemad-Moghadam, F. Tirgary, S. Keshavarz, and M. Alaeddini, "Head and neck non-Hodgkin's lymphoma: a 20-year demographic study of 381 cases," International Journal of Oral and Maxillofacial Surgery, vol. 39, no. 9, pp. 869872, 2010. 
[15] S. H. Swerdlow, E. Campo, N. L. Harris et al., WHO Classification of Tumours of Haematopoietic and Lymphoid Tissues, International Agency for Research on Cancer, Revised Fourth Edition, Lyon, France, 2017.

[16] P. A. Riedell and S. M. Smith, "Double hit and double expressors in lymphoma: definition and treatment," Cancer, vol. 124, no. 24, pp. 4622-4632, 2018.

[17] A. Rosenthal and A. Younes, "High grade B-cell lymphoma with rearrangements of MYC and BCL2 and/or BCL6: double hit and triple hit lymphomas and double expressing lymphoma," Blood Reviews, vol. 31, no. 2, pp. 37-42, 2017.

[18] M. Noujima-Harada, K. Takata, T. Miyata-Takata et al., "Frequent downregulation of BTB and CNC homology 2 expression in Epstein-Barr virus-positive diffuse large B-cell lymphoma," Cancer Science, vol. 108, no. 5, pp. 1071-1079, 2017.

[19] K. Fu, D. Weisenburger, W. W. Choi et al., "Addition of rituximab to standard chemotherapy improves the survival of both the germinal center B-cell-like and non-germinal center Bcell-like subtypes of diffuse large B-cell lymphoma," Journal of Clinical Oncology, vol. 26, no. 28, pp. 4587-4594, 2008.

[20] Z. Fu, J. Zhu, Y. Song, W. Liu, X. Ji, and S. Zhan, "Prognostic analysis of 525 Chinese patients with diffuse large B cell lymphoma," Journal of Peking University Health Science, vol. 46, no. 3, pp. 405-411, 2014.

[21] M. S. Cairo, R. Sposto, M. Gerrard et al., "Advanced stage, increased lactate dehydrogenase, and primary site, but not adolescent age ( $\geq 15$ years), are associated with an increased risk of treatment failure in children and adolescents with mature B-cell non-Hodgkin's lymphoma: results of the FAB LMB 96 study," Journal of Clinical Oncology, vol. 30, no. 4, pp. 387-393, 2012.

[22] S. Kang, H. Cho, B. S. Sohn et al., "Long-term follow-up of abbreviated R-CHOP chemoimmunotherapy for completely resected limited-stage diffuse large B cell lymphoma (CISL 12-09)," Annals of Hematology, vol. 99, no. 12, pp. 28312836, 2020.

[23] R. I. F. Van der Waal, P. C. Huijgens, P. Van der Valk, and I. Van der Waal, "Characteristics of 40 primary extranodal non-Hodgkin lymphomas of the oral cavity in perspective of the new WHO classification and the international prognostic index," International Journal of Oral and Maxillofacial Surgery, vol. 34, no. 4, pp. 391-395, 2005.

[24] S. M. Cohen, M. Petryk, M. Varma, P. S. Kozuch, E. D. Ames, and M. L. Grossbard, "Non-Hodgkin's lymphoma of mucosaassociated lymphoid tissue," The Oncologist, vol. 11, no. 10, pp. 1100-1117, 2006.

[25] M. Kojima, N. Nakamura, K. Shimizu et al., "Histopathological variation of primary mucosa-associated lymphoid tissue lymphoma of the oral cavity," Pathology Oncology Research, vol. 13, no. 4, pp. 345-349, 2007.

[26] Non-Hodgkin's Lymphoma Classification Project, "A clinical evaluation of the International Lymphoma Study Group classification of non-Hodgkin's Lymphoma," Blood, vol. 89, no. 11, pp. 3909-3918, 1997.

[27] R. I. Fisher, M. LeBlanc, O. W. Press, D. G. Maloney, J. M. Unger, and T. P. Miller, "New treatment options have changed the survival of patients with follicular lymphoma," Journal of Clinical Oncology, vol. 23, no. 33, pp. 8447-8452, 2005.

[28] A. Freedman, "Follicular lymphoma: 2018 update on diagnosis and management," American Journal of Hematology, vol. 93, no. 2, pp. 296-305, 2018.
[29] R. Schmitz, R. M. Young, M. Ceribelli et al.et al., "Burkitt lymphoma pathogenesis and therapeutic targets from structural and functional genomics," Journal of Pediatric and Adolescent Gynecology, vol. 28, pp. e105-e107, 2015.

[30] V. Banthia, A. Jen, and A. Kacker, "Sporadic Burkitt's lymphoma of the head and neck in the pediatric population," International Journal of Pediatric Otorhinolaryngology, vol. 67, no. 1, pp. 59-65, 2003.

[31] M. R. Abdelwahed Hussein, "Non-Hodgkin's lymphoma of the oral cavity and maxillofacial region: a pathologist viewpoint," Expert Review of Hematology, vol. 11, no. 9, pp. 737-748, 2018.

[32] X. Li, G. Li, Z. Gao, X. Zhou, and X. Zhu, "The relative frequencies of lymphoma subtypes in China: a nationwide study of 10002 cases by the Chinese Lymphoma Study Group (CLSG)," Annals of Oncology, vol. 22, suppl 4, p. 141, 2011.

[33] J. Xiong and W. Zhao, "Advances in multiple omics of naturalkiller/T cell lymphoma," Journal of Hematology \& Oncology, vol. 11, no. 1, p. 134, 2018.

[34] C. P. Fox, M. Civallero, Y. H. Ko et al., "Survival outcomes of patients with extranodal natural-killer T-cell lymphoma: a prospective cohort study from the international T-cell Project," Lancet Haematology, vol. 7, no. 4, pp. e284-e294, 2020.

[35] S. Wang, P. Liu, L. Lu, W. Xu, X. Liu, and Y. Liu, "Extranodal NK/T cell lymphoma nasal type: a clinicopathological analysis," Chinese Journal of Clinical and Experimental Pathology, vol. 36, pp. 529-533, 2020.

[36] X. He, C. Tian, D. Yang, X. Xie, and H. Liu, "Analysis on clinicopathologic characteristics of 216 primary extranodular non-Hodgkin's lymphoma in head and neck," Journal of Clinical otorhinolaryngology Head and Neck Surgery, vol. 23, pp. 878-880, 2009.

[37] G. Gualco, P. Domeny-Duarte, L. Chioato, G. Barber, Y. Natkunam, and C. E. Bacchi, "Clinicopathologic and molecular features of 122 Brazilian cases of nodal and extranodal NK/T-cell lymphoma, nasal type, with EBV subtyping analysis," The American Journal of Surgical Pathology, vol. 35, no. 8, pp. 1195-1203, 2011.

[38] Y. Huang, S. Chen, R. Wei et al., "CD20-positive extranodal NK/T cell lymphoma: clinicopathologic and prognostic features," Virchows Archiv, vol. 477, no. 6, pp. 873-883, 2020.

[39] B. Wang and X. Hong, "Clinical characteristic and current status of treatment for nasal NK/T cell lymphoma," China Oncology, vol. 16, pp. 672-674, 2006.

[40] A. Picard, C. Cardinne, Y. Denoux, I. Wagner, F. Chabolle, and C. A. Bach, "Extranodal lymphoma of the head and neck: a 67case series," European Annals of Otorhinolaryngology, Head and Neck Diseases, vol. 132, no. 2, pp. 71-75, 2015.

[41] M. B. Moller, N. T. Pedersen, and B. E. Christensen, "Diffuse large B-cell lymphoma: clinical implications of extranodal versus nodal presentation-a population-based study of 1575 cases," British Journal of Haematology, vol. 124, no. 2, pp. 151-159, 2004.

[42] T. D. Silva, C. B. Ferreira, G. B. Leite, J. R. de Menezes Pontes, and H. S. Antunes, "Oral manifestations of lymphoma: a systematic review," Ecancermedicalscience, vol. 10, p. 665, 2016.

[43] R. Lo, S. Mohamad, M. Krishnamoorthy, I. Mohamad, S. Zulkarnain, and F. A. Hussain, "Mantle cell lymphoma: a rare vallecular tumour," Medeniyet Medical Journal, vol. 35, pp. 67-70, 2020. 